\title{
Doctor gratitude: a framework and practical suggestions
}

$\mathrm{S}$ arah was depressed. After being fired for a minor indiscretion, she was unable to find work. Sarah confided in her doctor, BA (one of the authors), who encouraged her to explain early in her next interview what had happened at her last job and how skilled she is at her work. Sarah took his advice and was able to find a job. Her gratitude for this advice made BA grateful to have been in a position to help her.

What motivates a physician? Financial reward programs risk undermining the motivation within doctors to engage in challenging activities. Could doctors' gratitude for their work inspire their performance and reclaim authentic meaning for medicine?

Doctors are often silent on their own gratitude. In a growing culture of entitlement, they seldom openly discuss their gratitude for the opportunity to practise medicine. They tend to be silent about competing values that may not balance easily. For example, pride can act against gratitude, and doctors might not feel gratitude if they attribute their professional standing to luck, fate or largely their own doing; do not recognize medicine as a service industry; and hesitate to re-attach their work to their emotions.

Meanwhile, society grants doctors the gift, privilege and responsibility to practise medicine. Doctors enjoy material benefits and high social status, and can profit from altruism toward their patients. Doctors' gratitude is needed for the sake of their humility, to help anchor control and meaning in their work, and to moderate the negative effects of materialistic strivings. ${ }^{2}$ Thus, although doctors cannot be duty-bound to feel gratitude, they have a moral duty to think about, and seek to understand, the benefits of being a doctor. Through moral reflection and self-awareness they can habituate gratitude. ${ }^{3}$ Morally important for its own sake, gratitude can add joy and meaning to their work. It can strengthen doctors' social ties and commitment to generous helping and compassion, and help to meet their psychological needs for auton-

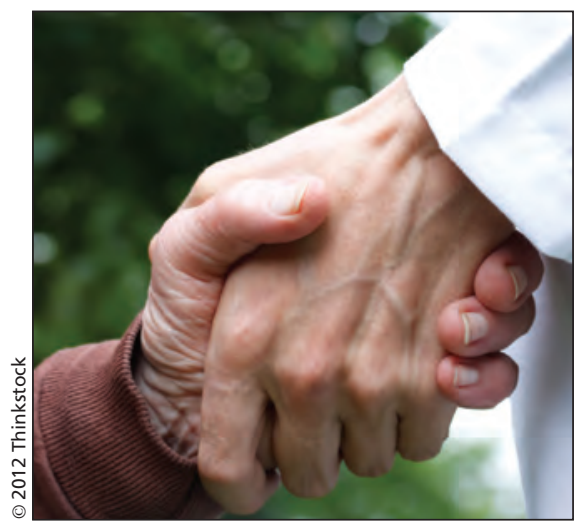

omy, competence and connectedness. ${ }^{4}$ As a form of social capital, it can support a contribution-based morality ${ }^{5}$ that adds balance to the reciprocal relations.

Doctors can show their gratitude through giving, receiving appropriate giving, and taking morally what has not been explicitly given to them. Although interrelated and not specific to gratitude, these categories frame how gratitude adds meaning to the work of doctors.

Giving by the doctor, especially when altruistic, can indicate and produce gratitude in the doctor (and patient). Altruism does not preclude self-benefit if the giving is not overwhelming. For example BA tries to attend patients' funerals. He does not do this for himself, but, in reminding himself and others how grateful he is to have known and cared for the patient, it makes him feel good.

In general, doctors can show and increase their gratitude through how they receive what others give them. Gratitude is indicated through actions taken - such as a smile or exhibiting patience - or not taken - such as resisting a temptation to act against patients' informed wishes. Doctor gratitude is also implicit when the doctor, rather than a designate, receives what a patient gives, including access to private spaces, such as patient homes.

In three circumstances doctors can feel and show gratitude in morally taking what has not been explicitly given to them. First, what the doctor takes has value and is possessable, but does not belong to anyone else, producing no cost to others. For example doctors are free to feel gratitude, to have a positive life orientation and to be satisfied in their work. BA is grateful that one of his difficult patients feels able to share her problems with him, which helps him cope with her visits. BA records his gratitude in a diary; one such entry depicts his gratitude at having acknowledged a medical student who subsequently died.

Second, a doctor can feel and show gratitude in taking actions with which patients can be reasonably expected to agree, had it been appropriate to ask them or if they had been able to approve. For example, to comfort a distressed patient, a doctor who touches their hand will not ask permission, and a doctor may treat an unconscious patient in a medical emergency on the presumption of patient consent.

Third, doctors can show gratitude for ideals that form the foundation for their actions when systems are in place to help them uphold these ideals in their practice. For example, doctors in Israel may access a legislative mechanism to uphold the sanctity of life.

Lying at the heart of what it means to be a doctor, gratitude can motivate doctors' work from within. It can help doctors balance the growing application of a business mindset to medicine. Grateful doctors feel good about themselves, feel connected to others and enjoy serving people who need them.

\section{Stephen A. Buetow PhD \\ Associate Professor \\ Bruce Aroll MBChB PhD \\ Professor \\ Department of General Practice and \\ Primary Health Care \\ University of Auckland \\ Auckland, New Zealand}

The character of Sarah in this work is fictitious. Any resemblance to real persons, living or dead, is purely coincidental.

For references, see Appendix 1, available at www.cmaj.ca/lookup/suppl/doi:10.1503 /cmaj.120422/-/DC1

CMAJ 2012. DOI:10.1503/cmaj.120422 